\title{
Household fish preparation hygiene and cholera transmission in Monrovia, Liberia
}

\author{
Pauline Scheelbeek ${ }^{1}$, Samuel Treglown ${ }^{1}$, Tony Reid ${ }^{2}$, Peter Maes ${ }^{3}$ \\ ${ }^{1}$ Public Health Specialist, Médecins Sans Frontières Belgium, Rue Dupre 94, 1070 Brussels, Belgium \\ ${ }^{2}$ Operational Research Centre, Médecins Sans Frontières Belgium, Rue Dupre 94, 1070 Brussels, Belgium \\ ${ }^{3}$ Coordinator of Water, Hygiene and Sanitation Unit; Médecins Sans Frontières Belgium, Rue Dupre 94, 1070 Brussels, Belgium
}

\begin{abstract}
Background: In the 1980s Vibrio cholerae was found to be an autochthonous resident of aquatic environments. As result, ingestion of undercooked, contaminated fish has been associated with cholera transmission. An alternative mechanism of transmission associated with fish was hypothesised by Schürmann et al. in 2002. He described a cholera case that was more likely to have been infected by contamination on the patient's hands rather than by ingestion of contaminated fish.

Methodology: With fish being the main diet in Liberia, we decided to examine fish samples and preparation techniques in Monrovia. Excreta of 15 fish, caught in the estuarine waters of Monrovia, were analysed for $V$. cholerae. In addition, fish preparation methods were observed in 30 households.

Results: Two fish samples were found positive. Observations revealed that hygiene measures during the gutting process of fish were limited; although hands were usually rinsed, in all cases soap was not used. Furthermore, contaminated water was frequently reused during food preparation.

Conclusions: Since the cooking process of fish (and thus elimination of bacteria) in Monrovia usually consists of both frying and boiling, it seems plausible that in this context, the hypothesis by Schürmann et al. could be applicable. Further research is necessary to confirm this association, which could be a starting point for more context-specific health education campaigns addressing food preparation hygiene as risk factor for cholera.
\end{abstract}

Keywords: Vibrio cholerae; fish preparation; hygiene; Liberia

J Infect Dev Ctries 2009; 3(9):727-731.

Received 22 February 2009 - Accepted 19 July 2009

Copyright $\odot 2009$ Scheelbeek et al. This is an open-access article distributed under the Creative Commons Attribution License, which permits unrestricted use, distribution, and reproduction in any medium, provided the original work is properly cited.

\section{Introduction}

While cholera still affects large numbers of people all over the world [1], its mechanism of transmission is not entirely clear. Previously, it was believed that Vibrio cholerae was solely linked to faecal contamination. However, studies from in the 1980s revealed that the micro-organism could occur in aquatic environments without the presence of faecal coliform bacteria [2,3,4]. Moreover, laboratory research supported the hypothesis that $V$. cholerae is an autochthonous resident of aquatic environments $[5,6]$ where it adheres to zooplankton and phytoplankton (algae).

Studies of the food chain in aquatic environments showed that smaller fish feed on plankton and could become infected with $V$. cholerae [7]. This led to the hypothesis that fish could be an important vehicle of cholera transmission to humans [8]. Furthermore, the micro-organism was found to survive for weeks in the stomach of fish, even when refrigerated [9]. Since fish has been eaten raw or undercooked in many countries, it has been associated with food-borne outbreaks of cholera [10] and has been shown to be the causative agent in affected patients [11].

However, the mechanisms by which $V$. cholerae is actually transmitted to humans remains unclear, particularly whether it is specifically by eating contaminated fish. In 2002, another possibility was raised by Schürmann et al. [11], while investigating a cholera case in Germany. He noticed that the interval between ingestion of a fish imported from Nigeria and the onset of symptoms was very short. Furthermore, the fish was cooked in boiling water, making it unlikely that the infection was caused specifically by eating the fish. A plausible alternative given by Schürmann et al. [11] was that the patient had contaminated his hands during the gutting 
Figure 1. Annual Case Numbers of Cholera Cases admitted to the Cholera Treatment Unit in Monrovia.

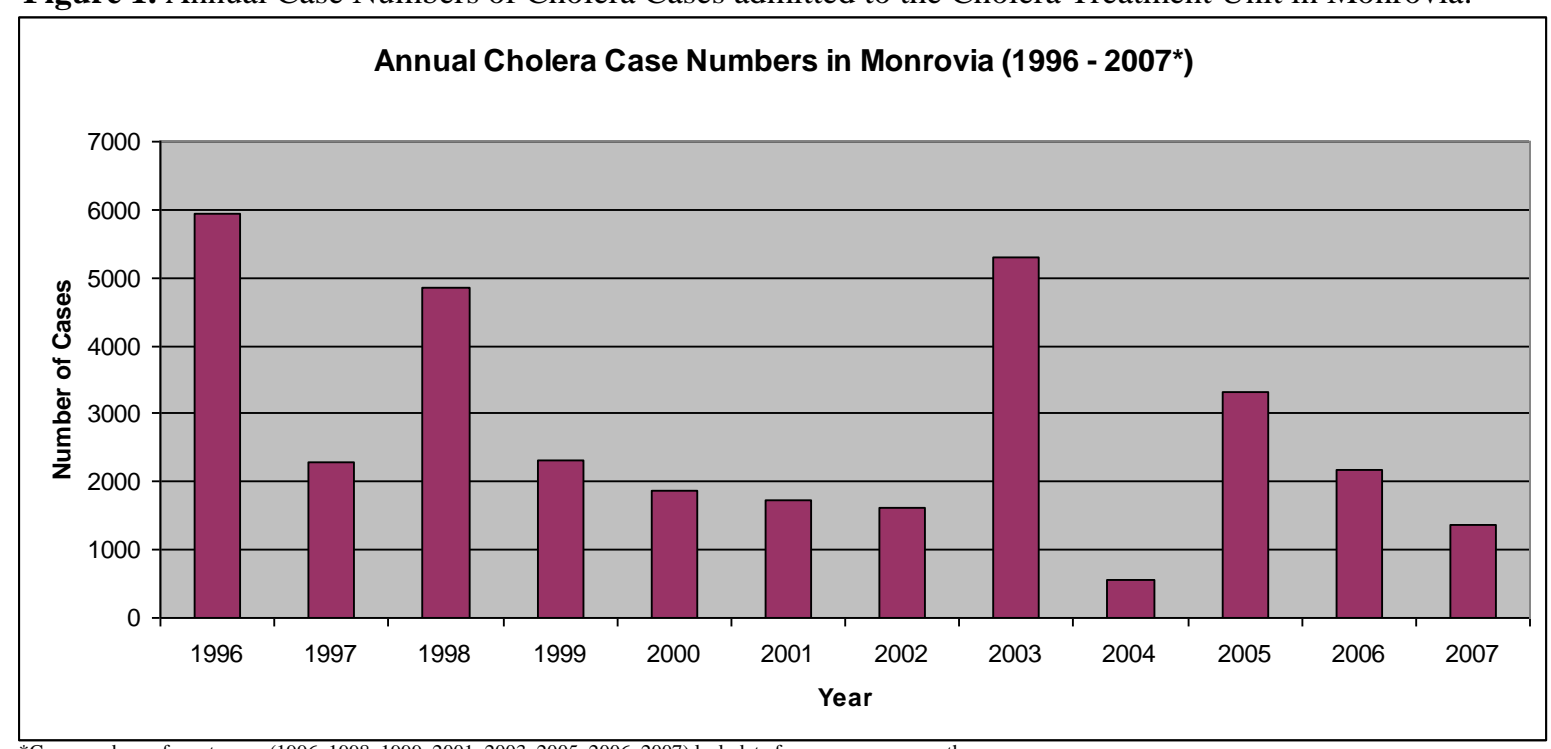

*Case numbers of most years $(1996,1998,1999,2001,2003,2005,2006,2007)$ lack data for one or more months

process and, as result of poor hand hygiene, had ingested the bacteria.

Monrovia has been affected by an increasing frequency of cholera outbreaks since the arrival of the ongoing seventh cholera pandemic in Africa [12]. In the last decade, regular cholera outbreaks have occurred, indicating that cholera is endemic in the country. Since the early 1990s Médècins Sans Frontières-Belgium (MSF-B) has been supporting the Liberian Ministry of Health by running a Cholera Treatment Unit (CTU) in Monrovia. Between 1996 and 2008 the case numbers admitted in this CTU ranged from 550 to almost 6000 cases. The case fatality rate was relatively low, not exceeding $1 \%$ between 2004 and 2007 (see Figure 1). This was, however, based on the admitted patients, which may be an underestimate of the real problem $[13,14]$.

In 2007 MSF-B conducted a study aimed at improving the understanding of cholera transmission in Monrovia. Food intake during the five days prior to onset of symptoms (the possible incubation period) was recorded from the patients admitted to the Ministry of Health/MSF-B CTU between March and August 2007; more than 91\% [95\% CI 85.2 - 97.5\%] had eaten fish within this period that had been prepared inside the household [15]. The geographically dispersed home locations of the patients admitted to the CTU suggested that noncentralised transmission routes were more significant than concentrated point sources of transmission [12]; therefore, we decided to have a closer look at the fish in the estuaries around Monrovia to see whether they could be a vehicle for cholera transmission and to examine fish preparation techniques. In Monrovia, fish is usually bought whole (or in cross-sectional slices), either at the market, from roaming fish-sellers walking through the city, or directly from fishermen. The fish are not cleaned before being sold, but taken home to be gutted by the household members themselves. Furthermore, the cooking process in Monrovia usually includes both frying and boiling of the fish. This combination of features suggested a possible transmission pattern based on cleaning the fish rather than its consumption.

Thus, the objective of this report is to describe a possible mechanism of transmission of $V$. cholerae through unhygienic food preparation in Monrovia, Liberia.

\section{Methods}

Analysis of fish samples

The coastal city of Monrovia is the home to a large market for fish and seafood products. Samples of fish, caught by Kru fishermen, were collected from each of the four main landing points for Kru fish boats in Monrovia: Banja beach, Popo beach, West Point peninsula and ELWA beach. At three beaches fish samples were bought directly from fishing boats after they had landed. At the other beach, unpredictable landing times forced us to buy fish samples from market vendors, who had purchased the fish from the boats that had landed earlier that day. 
A total of 15 samples were collected: 14 small fish (approximately $10-15 \mathrm{~cm}$ in length) and one was a cross-sectional slice of a larger fish. This number was small due to limited access to lab culture facilities. After purchase, the fish were wrapped in newspapers by the vendors/fishermen and individually placed in a sealed plastic bag. This bag was placed in a cool box with icepacks and was transported the same day to the MSF laboratory. In that laboratory the fish were unwrapped and an incision was made to expose the intestines. Then, the excreta were removed using a pipette and placed into a specimen jar. From there, the specimen jars were stored in a fridge overnight in the MSF laboratory (temperature $<8^{\circ} \mathrm{C}$ ). The next day they were transported in a cool box to the laboratory of Catholic Hospital in Monrovia for further analysis on $V$. cholerae.

On arrival at the Catholic Hospital, the specimens were inoculated onto Thiosulfate Citrate Bile Salts Sucrose Agar (TCBS Agar A, a selective medium for $V$. cholerae) and incubated overnight (18-24 hours) at a temperature of $35^{\circ} \mathrm{C}$. In addition, another preparation was made using Alkaline Peptone Water (APW, enrichment medium) and incubated for six hours at a temperature of $35^{\circ} \mathrm{C}$ to stimulate growth. This broth was then sub-cultured onto another TCBS agar (TCBS Agar B), which was also incubated overnight (18-24 hours) at a temperature of $35^{\circ} \mathrm{C}$. From TCBS agar B, serotyping was performed, using antisera (Biotec) for the rapid slide agglutination test. Due to the limited amounts of financial resources and laboratory equipment, the isolates were not subcultured on nutrient agar before serotyping was performed, which could possibly have led to contamination.

\section{Household observations}

To identify possible transmission of $V$. cholerae due to poor hygiene, household observations were conducted. This kind of community observation was usual practice for the MSF water and sanitation programme during cholera outbreaks. Six health districts (10.000 - 20.000 people) in Monrovia were selected for the observations. At each location the observation team visited four to seven households where fish preparation was taking place.

Selection of households was done by starting in the centre of the community and walking towards the main road. Wherever gutting was noticed, the people cleaning the fish and/or their parents were asked for permission to observe the gutting process and write down notes. Each observation session was recorded by two observers, who were local nationals, and information was cross-checked between them. Hygiene measures used during the fish gutting process were observed, including method and frequency of hand washing and disposal of guts after cleaning the fish. The observations were recorded on a tick list and, where necessary, questions were asked for clarification and interpretation of the observations. The process was observed from the start of the fish cleaning process until the start of cooking.

This report was approved by the MSF Ethics Review Board.

\section{Results}

\section{Fish Samples}

All fishermen who were approached agreed to give a sample fish. After overnight incubation, the A Agars showed a large number of Vibrio colonies for two out of 15 specimens (specimen \#3 <Banja Beach> and specimen \#4 <Popo Beach>). The other 13 specimens showed no growth. TCBS Agar B, (enriched with Alkaline Peptone Water), also showed a positive result for Vibrio in specimens \#3 and \#4. All samples were identified to be serotype 01 .

\section{Household Food Preparation Observation}

In the six chosen areas, all people who were approached agreed to being observed for a total of 30 households. We noted many similarities of food preparation hygiene regardless of differences in housing, religion, social status, etc. The following is a summary of the observations:

(1) All gutters used their hands for the removal of excreta.

(2) After removal of the excreta, $96 \%$ of the gutters did not wash their hands with soap. All gutters $(100 \%)$ rinsed their hands, mainly because fish scales were sticking to their hands.

(3) The wastewater generated by hand rinsing frequently fell into the bowl where the gutted fish was kept.

(4) After removal of the guts, all gutters threw the guts back into the bowl with the fish.

(5) The bowl and the knife used for gutting were rinsed by all gutters. However, the water used for rinsing was the dirty water from the bowl (where gutted fish, excreta and wastewater from hand washing was kept). 
(6) The disposal of the fish guts was often close to the house: $56 \%$ of the gutters emptied the bowl with water and excreta behind the house, while another $28 \%$ poured the wastewater and fish offal into a water body (such as river, sea or swamp).

(7) Only $7 \%$ were observed to bury the excreta and wastewater in a hole in the ground.

\section{Discussion}

The association between eating contaminated fish and clinical cholera has already been established, but the study reported here appears to suggest an alternative mechanism of transmission - that of unsanitary practices while cleaning and preparing fish. It supports the hypothesis that, besides ingestion, cholera can be transmitted via contaminated hands, as a result of poor hygiene practices during the gutting process. In Liberia, fish are usually fried and boiled, which minimises the chance of survival of $V$. cholerae in fish that are being eaten. Based on our observations, the practice of gutting the fish at home and not washing hands, cutlery or bowls that were subsequently used to serve the fish, would provide a credible way for $V$. cholerae to be transmitted and should be further investigated for its role and importance in cholera transmission in Monrovia..

Our report reinforces the conclusion of the German investigation that suggested contaminated preparation as a method of transmission [11]. It also adds evidence to the notion that cholera can be transmitted via the food chain in fish that are exposed to contaminated algae and plankton in polluted water $[716,17]$.

Although only two out of 15 fish were culture positive, they were positive in two separate tests, including one without Alkaline Peptone Water, which is normally used to amplify the result. This suggests that the fish were quite heavily contaminated and more likely to be a source of transmission.

These observations are important because in cities such as Monrovia, where cholera has become endemic, health and hygiene education messages must be accurate and context-specific. Emphasizing proper cooking of fish is unlikely to affect transmission in this context, since this is already practiced. However, if the preparation of fish is unsanitary, a new orientation in health promotion is needed, including messages emphasising:

(1) the potential hazard of offal and fish excreta

(2) the separation of excreta and gutted fish

(3) burial of excreta, instead of disposing it in the (aquatic) environment

(4) washing of hands, cutlery and pots with soap

There were a number of limitations to this report. It was an observational study conducted during regular cholera control activities by MSF and cannot prove causal evidence for this method of transmission of cholera. Only 15 fish could be tested for culture due to a limited availability of consumables at the Catholic Hospital laboratory where the microbiological analyses were performed. Therefore, a larger study needs to be done to confirm these findings using a larger sample of fish as well as further observations and cultures of fish handlers. In addition, the results given by the laboratory for the two positive cultures was " $V$. cholerae 01 ". Since the oxidase test was not performed in the laboratory, the colonies on the agar could be either $V$. cholerae or $V$. fluvialis serogroup. However, there is no documented V. fluvialis found in Monrovia, making it unlikely that the colonies are from the V. fluvialis serogroup. The serotyping was performed with the TBSC agar, where bile salts could have interfered with the results. Lastly, it should be noted that, due to old demographic data, the selection of four out of six observation districts was based on overall incidence of cholera in 2007, rather than exact incidence rates.

\section{Conclusion}

Despite its limitations, our report adds evidence to the mechanisms of cholera transmission. In addition to ingestion of cholera-infected fish, unsanitary practices during and after the fish-cleaning process may be contributing factors. A larger study should be done to confirm these findings. If unsanitary preparation of fish is proven to be an important vehicle for cholera, proper methods of food preparation hygiene should be considered as part of the health promotion campaign.

\section{Acknowledgements}

The authors would like to thank Patrick Laurent for the initial investigations; Mary Toe and John Keller for the data collection in the communities; and Tanja Thurn, Winnifred Yah Hallowanger and Gaby Rammersmeier for their assistance in the laboratory. 


\section{References}

1. Griffith D, Kelly-Hope L, Miller M (2006) Review of reported cholera outbreaks worldwide, 1995-2005. Am J Trop Med Hyg 75: 973-977.

2. Colwell R, Seidler R, Kaper J, Joseph S, Garges S, Lockman H, Maneval D, Bradford H, Roberts N, Remmers E, Huq I, Huq A (1981) Occurrence of Vibrio cholerae serotype 01 in Maryland and Louisiana estuaries. Appl Environ Microbiol 41: 555-558.

3. Hood M, Ness G, Rodrick G (1981) Isolation of Vibrio cholerae serotype 01 from the eastern oyster, Crassostrea virginica. Appl Environ Microbiol 41: 559-560.

4. Hood M, Ness G (1982) Survival of Vibrio cholerae and Escherichia coli in estuarine waters and sediments. Appl Environ Microbiol 43: 578-584.

5. Miller C, Drasar B, Feachem R (1984) Response of toxogenic Vibrio cholerae 01 to physico-chemical stress in aquatic environments. J Hyg (Lond) 93: 475-495

6. Singleton F, Attwell R, Jangi M, Colwell R (1982) Influence of salinity and organic nutrient concentration on survival and growth of Vibrio cholerae in aquatic microcosms. Appl Environ Microbiol 43: 1080-1085.

7. Huq A, Small E, West P, Huq M, Rahman R, Colwell R (1983) Ecological relationships between Vibrio cholerae and planktonic crustacean copepods. Appl Environ Microbiol 45: 275-283.

8. World Health Organisation (1952) Agreement for assistance in connexion with the carrying out of epidemiological investigations in cholera. New Delhi, India.

9. DePaola A (1981) Vibrio cholerae in marine foods and environmental waters: literature review. J. Food Sci 46: 6670.

10. Rabbani G, Greenough W (1999) Food as Vehicle Transmission of Cholera. J Diarrhoeal Ds Res Mar 17: 1-9.

11. Schürmann D, Ebert N, Kampf D, Baumann B, Frei U, Suttorp N (2002) Domestic Cholera in Germany Associated with Fresh Fish Imported from Nigeria. Eur J Clin Microbiol Infect Dis 21: 827-828.

12. Treglown S (2007) Report on weeks 12-16, 2007 of MSF Belgium cholera study and intervention, Monrovia, Liberia. MSF internal document.

13. Médecins Sans Frontières (2008) Report on the 2007 cholera study in Monrovia, Liberia. MSF internal document

14. Médecins Sans Frontières (2008) Cholera Surveillance Report, 2008. MSF internal document.

15. Treglown S, Scheelbeek P (2008) Report on weeks 12-31, 2007 of MSF Belgium cholera study and intervention, Monrovia, Liberia. MSF internal document

16. Islam M, Drasar B, Bradley D (1989) Attachment of toxogenic Vibrio cholerae 01 to various fresh-water plants and survival with a filamentous green alga, Rhizoclonium fontanum. J Trop Med Hyg 92: 396-401.

17. Tamplin M, Gauzens A, Huq A, Sack R, Colwell R (1990) Attachment of Vibrio cholerae serogroup 01 to zooplankton and phytoplankton of Bangladesh waters. Appl Environ Microbiol 56: 1977-1980.

\section{Corresponding author}

Pauline Scheelbeek

Médecins Sans Frontières Belgium

Rue Dupre 94

1070 Brussels, Belgium

Tel: +31.620112747

Email: Pauline.Scheelbeek@gmail.com

Conflict of Interest: No conflict of interest is declared. 